CIRJE-F-787

\title{
A General Computation Scheme for a High-Order Asymptotic Expansion Method
}

\author{
Akihiko Takahashi \\ University of Tokyo \\ Kota Takehara \\ Graduate School of Economics, University of Tokyo \\ Masashi Toda \\ Graduate School of Economics, University of Tokyo \\ February 2011; February and October 2012
}

CIRJE Discussion Papers can be downloaded without charge from:

http://www.cirje.e.u-tokyo.ac.jp/research/03research02dp.html

Discussion Papers are a series of manuscripts in their draft form. They are not intended for circulation or distribution except as indicated by the author. For that reason Discussion Papers may not be reproduced or distributed without the written consent of the author. 


\title{
A General Computation Scheme for a High-Order Asymptotic Expansion Method *
}

\author{
Akihiko Takahashi, Kohta Takehara ${ }^{\dagger}$ and Masashi Toda ${ }^{\ddagger}$ \\ Graduate School of Economics, the University of Tokyo \\ 7-3-1, Hongo, Bunkyo-ku, Tokyo, 113-0033, Japan, +81-3-3812-2111
}

First Version: May 6, 2010, Final Version: February 10, 2012

\begin{abstract}
This paper presents a new computational scheme for an asymptotic expansion method of an arbitrary order.

The asymptotic expansion method in finance initiated by Kunitomo and Takahashi [9], Yoshida [34] and Takahashi [20], [21] is a widely applicable methodology for an analytic approximation of expectation of a certain functional of diffusion processes. Hence, not only academic researchers but also many practitioners have used the methodology for a variety of financial issues such as pricing or hedging complex derivatives under high-dimensional underlying stochastic environments. In practical applications of the expansion, a crucial step is calculation of conditional expectations for a certain kind of Wiener functionals. [20], [21] and Takahashi and Takehara [23] provided explicit formulas for those conditional expectations necessary for the asymptotic expansion up to the third order.

This paper presents the new method for computing an arbitrary-order expansion in a general diffusion-type stochastic environment, which is powerful especially for high-order expansions: We develops a new calculation algorithm for computing coefficients of the expansion through solving a system of ordinary differential equations that is equivalent to computing the conditional expectations directly. To demonstrate its effectiveness, the paper gives numerical examples of the approximation for a $\lambda$-SABR model up to the fifth order.
\end{abstract}

Keywords: Asymptotic Expansion, Malliavin Calculus, Approximation Formula, Stochastic Volatility, $\lambda$-SABR Model

AMS Subject Classifications: 91G80, 91G20, 60H07, 60H30, 60H35

\footnotetext{
${ }^{*}$ This is partially supported by the global COE program "The research and training center for new development in mathematics" and Grant-in-Aid for Research Fellow of JSPS.

†fin.tk.house@gmail.com. Research Fellow of the Japan Society for the Promotion of Science(JSPS).

${ }^{\ddagger}$ Research Fellow of the Japan Society for the Promotion of Science(JSPS).
} 


\section{Introduction}

This paper presents a new scheme for computation in the method so-called "an asymptotic expansion approach" by developing a new calculation algorithm.

To our best knowledge, the 'asymptotic expansion method' was firstly introduced to a financial literature by [9] and [20] with an application to the evaluation of an average option that is a popular derivative in commodity markets. They derive the approximation formulas for the average option by the asymptotic expansion method based on log-normal approximations of a distribution of an average price when an underlying asset price follows a geometric Brownian motion. [34] applies a formula derived through the asymptotic expansion of certain statistical estimators for small diffusion processes to approximating average option prices. Thereafter, the asymptotic expansion have been applied to a broad class of problems in finance: See [21], [22], Kunitomo and Takahashi [10], [11], Matsuoka, Takahashi and Uchida [14], Takahashi and Yoshida [30], [31], Muroi [15], and Takahashi and Takehara [23], [24], [25]. It is notable that the method has flexible applicability to a broad class of diffusion-type stochastic settings in a unified way, and mathematical justification by Watanabe theory(Watanabe [32], Yoshida [33]) in Malliavin calculus.

There are also other various approaches for approximation of solutions to pricing PDEs, Greeks and heat kernels through certain asymptotic expansions: for instance, there are recent works such as Fouque, Papanicolaou and Sircar [4], [5], Hagan, Kumar, Lesniewski and Woodward [7], Henry-Labordere [12], [13], Siopacha and Teichmann [19], Ben Arous and Laurence [3] and Gatheral, Hsu, Laurence, Ouyang and Wang [6].

Recently, not only academic researchers but also many practitioners such as Antonov and Misirpashaev [1] or Andersen and Hutchings [2] have used the asymptotic expansion method based on Watanabe theory in or combined with their techniques for a variety of financial issues. e.g. pricing or hedging complex derivatives under high-dimensional underlying stochastic environments. These methods fully or partially rely on the framework developed by [9], [20], [21] in a financial literature.

In theory, this method provides us the expansion, which has a proper meaning in the limit of some ideal situations such as cases where these processes would be deterministic, of underlying stochastic processes (for the detail see [32], [33] or [11]).

In practice, however, we are often interested in cases far from those situations, where the underlying processes are highly volatile as seen in recent financial markets especially after the crisis on 2008. Then from view points of accuracy or stability of the techniques in practical uses, it is desirable to investigate behaviors of its estimators especially with expansion up to high orders in such environments.

In the existing application of the asymptotic expansion based on Watanabe theory, they calculated certain conditional expectations which appear in their expansions and which play key roles in computation, by formulas up to the third order given explicitly in [20], [21] and [23]. In many applications, these formulas give sufficiently accurate approximation, but in some cases, for example in cases with long maturities or/and with highly volatile underlying variables, the approximations up to the third order may not provide satisfactory accuracies. Thus, formulas for higher-order computations are desirable. But to our knowledge, the asymptotic expansion formulas higher than the third order in a general setting have not been given yet.

This paper provides a new scheme for computing unconditional expectations which is completely equivalent to direct calculation of the conditional expecta- 
tions. This enables us to derive the high-order approximation formulas in an automatic manner. Consequently, our approximation generally shows sufficient accuracy in computation of high-order expansions, which is confirmed by numerical experiments. In those experiments, comparing our method to another existing one, we also see its advantageous applicability in financial practice.

Recently, Takehara, Takahashi and Toda [27] introduced a computational scheme for the conditional expectations as well as an equivalent scheme for the unconditional ones in the same manner to this work, and gave some formulas for the fourth order expansion. That paper and Takehara, Toda and Takahashi [28] applied those to option pricing in the $\lambda$-SABR model and in a cross-currency setting with long maturities. However, in those papers key ideas of the methods were heuristically shown only in a very simple setting while their applications were out of that framework. Thus, in this sense, this paper can be considered to give justification and generalization of those works to a much broader class of models.

Organization of this paper is as follows: After a brief explanation of the asymptotic expansion in Section 2, Section 3 introduces our new computation algorithm and derives the formulas for the asymptotic expansion. Section 4 applies our algorithm described in the previous section to the concrete financial models, and confirms effectiveness of the high-order expansions by numerical examples in the $(\lambda-)$ SABR model. Finally, Appendix shows proofs of Lemma 1 and Theorem 1 in Section 3, which are omitted in the main text. Due to the limitation of space, some related results and some of concrete data of the experiments in Section 4 are omitted and left in our online working paper CARF-F-149 [26] (http://www.carf.e.u-tokyo.ac.jp/pdf/workingpaper/fseries/154.pdf).

\section{An Asymptotic Expansion in a General Dif- fusion Setting}

This section briefly describes an asymptotic expansion method in a general diffusion setting.

Let $(W, P)$ be a $r$-dimensional Wiener space. We consider a $d$-dimensional diffusion process $X_{t}^{(\epsilon)}=\left(X_{t}^{(\epsilon), 1}, \cdots, X_{t}^{(\epsilon), d}\right)^{\prime}$ which is the solution to the following stochastic differential equation:

$$
\begin{aligned}
& d X_{t}^{(\epsilon), j}=V_{0}^{j}\left(X_{t}^{(\epsilon)}, \epsilon\right) d t+\epsilon V^{j}\left(X_{t}^{(\epsilon)}\right) d W_{t} \quad(j=1, \cdots, d) \\
& X_{0}^{(\epsilon)}=x_{0} \in \mathbf{R}^{d}
\end{aligned}
$$

where $W=\left(W^{1}, \cdots, W^{r}\right)^{\prime}$ is a $r$-dimensional standard Wiener process, and $\epsilon \in(0,1]$ is a known parameter.

Suppose that $V_{0}=\left(V_{0}^{1}, \cdots, V_{0}^{d}\right)^{\prime}: \mathbf{R}^{d} \times(0,1] \mapsto \mathbf{R}^{d}$ and $V=\left(V^{1}, \cdots, V^{d}\right)$ : $\mathbf{R}^{d} \mapsto \mathbf{R}^{d} \otimes \mathbf{R}^{r}$ satisfy some regularity conditions(for example, $V_{0}$ and $V$ are smooth functions with bounded derivatives of all orders).

Next, let a function $g: \mathbf{R}^{d} \mapsto \mathbf{R}$ be smooth and all of its derivatives have polynomial growth. Then, a smooth Wiener functional $g\left(X_{T}^{(\epsilon)}\right)$ has its asymptotic expansion:

$$
g\left(X_{T}^{(\epsilon)}\right) \sim g_{0 T}+\epsilon g_{1 T}+\epsilon^{2} g_{2 T}+\cdots
$$

in $\mathbf{D}^{\infty}$ as $\epsilon \downarrow 0$ where $g_{0 T}, g_{1 T}, g_{2 T}, \cdots \in \mathbf{D}^{\infty}$. For any $k \in \mathbf{N}, q \in(1, \infty)$ and $s>0$, this expansion means that

$$
\frac{1}{\epsilon^{k}}\left\|g\left(X_{T}^{(\epsilon)}\right)-\left(g_{0 T}+\epsilon g_{1 T}+\cdots+\epsilon^{k-1} g_{k-1, T}\right)\right\|_{q, s}=O(1)(\text { as } \epsilon \downarrow 0),
$$


where $\|G\|_{q, s}$ represents the sum of $L^{q}$-norms of Malliavin derivatives of a Wiener functional $G$ up to the $s$-th order. Further, a Banach space $\mathbf{D}_{q, s}=$ $\mathbf{D}_{q, s}(\mathbf{R})$ can be regarded as the totality of random variables bounded with respect to $(q, s)$-norm $\|\cdot\|_{q, s}$, and $\mathbf{D}^{\infty}=\cap_{s>0} \cap_{1<q<\infty} \mathbf{D}_{q, s}$.

Coefficients $g_{n T} \in \mathbf{D}^{\infty}(n=0,1, \cdots)$ in the expansion can be obtained by Taylor's formula and represented based on multiple Wiener-Itô integrals. See chapter $\mathrm{V}$ of Ikeda and Watanabe [8] for the detail.

In financial applications, for example $X^{(\epsilon)}$ consists of two stocks, $X^{(\epsilon)}=$ $\left(S_{1}^{(\epsilon)}, S_{2}^{(\epsilon)}\right)^{\prime}$ and $g(\cdot)$ is their average $g(x)=\frac{x_{1}+x_{2}}{2}$ for $x=\left(x_{1}, x_{2}\right)^{\prime}$ : As another example, we can set $X^{(\epsilon)}$ is a vector of $N$ discrete Libor forward rates, $X^{(\epsilon)}=$ $\left(L_{1}^{(\epsilon)}, \cdots, L_{N}^{(\epsilon)}\right)^{\prime}$, and

$$
g\left(X_{T}^{(\epsilon)}\right)=S R_{T}^{(\epsilon)}=\frac{1-\prod_{j=1}^{N} \frac{1}{1+\tau L_{j T}^{(\epsilon)}}}{\tau \sum_{i=1}^{N} \prod_{j=1}^{i} \frac{1}{1+\tau L_{j T}^{(\epsilon)}}},
$$

that is a swap rate with maturity $T_{N}=T+N \tau$.

Let $A_{k t}=\left.\frac{1}{k !} \frac{\partial^{k} X_{t}^{(\epsilon)}}{\partial \epsilon^{k}}\right|_{\epsilon=0}$ and $A_{k t}^{j}, j=1, \cdots, d$ denote the $j$-th elements of $A_{k t}$. In particular, $A_{1 t}$ is represented by

$$
A_{1 t}=\int_{0}^{t} Y_{t} Y_{u}^{-1}\left(\partial_{\epsilon} V_{0}\left(X_{u}^{(0)}, 0\right) d u+V\left(X_{u}^{(0)}\right) d W_{u}\right)
$$

where $Y$ denotes the solution to the ordinary differential equation:

$$
d Y_{t}=\partial V_{0}\left(X_{t}^{(0)}, 0\right) Y_{t} d t ; Y_{0}=I_{d} .
$$

Here, $\partial V_{0}$ denotes the $d \times d$ matrix whose $(j, k)$-element is $\partial_{k} V_{0}^{j}=\frac{\partial V_{0}^{j}(x, \epsilon)}{\partial x_{k}}, V_{0}^{j}$ is the $j$-th element of $V_{0}$, and $I_{d}$ denotes the $d \times d$ identity matrix.

For $k \geq 2, A_{k t}^{j}, j=1, \cdots, d$ is recursively determined by the following equation:

$$
\begin{aligned}
A_{k t}^{j}= & \frac{1}{k !} \int_{0}^{t} \partial_{\epsilon}^{k} V_{0}^{j}\left(X^{(0)}, 0\right) d u \\
& +\sum_{l=1}^{k} \sum_{\vec{l}_{\beta}, \vec{d}_{\beta}}^{(l)} \frac{1}{(k-l) !} \frac{1}{\beta !} \int_{0}^{t}\left(\prod_{j=1}^{\beta} A_{l_{j} u}^{d_{j}}\right) \partial_{\vec{d}_{\beta}}^{\beta} \partial_{\epsilon}^{k-l} V_{0}^{j}\left(X_{u}^{(0)}, 0\right) d u \\
& +\sum_{\vec{l}_{\beta}, \vec{d}_{\beta}}^{(k-1)} \frac{1}{\beta !} \int_{0}^{t}\left(\prod_{j=1}^{\beta} A_{l_{j} u}^{d_{j}}\right) \partial_{\vec{d}_{\beta}}^{\beta} V^{j}\left(X_{u}^{(0)}\right) d W_{u}
\end{aligned}
$$

where $\partial_{\epsilon}^{l}=\frac{\partial^{l}}{\partial \epsilon^{l}}, \partial_{\vec{d}_{\beta}}^{\beta}=\frac{\partial^{\beta}}{\partial x_{d_{1}} \cdots \partial x_{d_{\beta}}}$,

$$
L_{n, \beta}=\left\{\vec{l}_{\beta}=\left(l_{1}, \cdots, l_{\beta}\right) ; \sum_{j=1}^{\beta} l_{j}=n, l_{j} \geq 1, j=1, \cdots, \beta\right\}
$$

and

$$
\sum_{\vec{l}_{\beta}, \vec{d}_{\beta}}^{(n)}=\sum_{\beta=1}^{n} \sum_{\vec{l}_{\beta} \in L_{n, \beta}} \sum_{\vec{d}_{\beta} \in\{1, \cdots, d\}^{\beta}}
$$


for $n \geq 1$, and

$$
\sum_{\vec{l}_{\beta}, \vec{d}_{\beta}}^{(0)}=\sum_{\beta=0} \sum_{\vec{l}_{0}=(\emptyset)} \sum_{\vec{d}_{0}=(\emptyset)} .
$$

Then, $g_{0 T}$ and $g_{1 T}$ can be written as

$$
\begin{aligned}
& g_{0 T}=g\left(X_{T}^{(0)}\right), \\
& g_{1 T}=\sum_{j=1}^{d} \partial_{j} g\left(X_{T}^{(0)}\right) A_{1 T}^{j} .
\end{aligned}
$$

For $n \geq 2, g_{n T}$ is expressed as follows:

$$
g_{n T}=\sum_{\overrightarrow{l_{\beta}}, \overrightarrow{d_{\beta}}}^{(n)} \frac{1}{\beta !} \partial_{\vec{d}_{\beta}}^{\beta} g\left(X_{T}^{(0)}\right) A_{l_{1} T}^{d_{1}} \cdots A_{l_{\beta} T}^{d_{\beta}} .
$$

Next, let normalize $g\left(X_{T}^{(\epsilon)}\right)$ to

$$
G^{(\epsilon)}=\frac{g\left(X_{T}^{(\epsilon)}\right)-g_{0 T}}{\epsilon}
$$

for $\epsilon \in(0,1]$. Then, we have

$$
G^{(\epsilon)} \sim g_{1 T}+\epsilon g_{2 T}+\cdots
$$

in $\mathbf{D}^{\infty}$. Moreover, let

$$
\hat{V}(x, t)=(\partial g(x))^{\prime}\left[Y_{T} Y_{t}^{-1} V(x)\right]
$$

and make the following assumption:

$$
\text { (Assumption 1) } \Sigma_{T}=\int_{0}^{T} \hat{V}\left(X_{t}^{(0)}, t\right) \hat{V}\left(X_{t}^{(0)}, t\right)^{\prime} d t>0 .
$$

Note that $g_{1 T}$ follows a normal distribution with variance $\Sigma_{T}$; the density function of $g_{1 T}$ denoted by $f_{g_{1 T}}(x)$ is given by

$$
f_{g_{1 T}}(x)=\frac{1}{\sqrt{2 \pi \Sigma_{T}}} \exp \left(-\frac{(x-C)^{2}}{2 \Sigma_{T}}\right)
$$

where

$$
C:=\left(\partial g\left(X_{T}^{(0)}\right)\right)^{\prime} \int_{0}^{T} Y_{T} Y_{t}^{-1} \partial_{\epsilon} V_{0}\left(X_{t}^{(0)}, 0\right) d t .
$$

Hence, Assumption 1 means that the distribution of $g_{1 T}$ does not degenerate. In application, it is easy to check this condition in most cases. Hereafter, Let $\mathcal{S}$ be the real Schwartz space of rapidly decreasing $\mathbf{C}^{\infty}$-functions on $\mathbf{R}$ and $\mathcal{S}^{\prime}$ be its dual space that is the space of the Schwartz tempered distributions. Next, take $\Phi \in \mathcal{S}^{\prime}$. Then, by Watanabe theory (Watanabe [32], Yoshida [33]) a generalized Wiener functional $\Phi\left(G^{(\epsilon)}\right)$ has an asymptotic expansion in $\mathbf{D}^{-\infty}$ as $\epsilon \downarrow 0$ where $\mathbf{D}^{-\infty}$ denotes the set of generalized Wiener functionals. See chapter V of Ikeda and Watanabe [8] for the detail. Hence, the expectation of $\Phi\left(G^{(\epsilon)}\right)$ is expanded 
around $\epsilon=0$ as follows: For $N=0,1,2, \cdots$,

$$
\begin{aligned}
\mathbf{E}\left[\Phi\left(G^{(\epsilon)}\right)\right] & =\sum_{n=0}^{N} \epsilon^{n} \sum_{\vec{k}_{\delta}}^{(n)} \frac{1}{\delta !} \mathbf{E}\left[\Phi^{(\delta)}\left(g_{1 T}\right)\left(\prod_{j=1}^{\delta} g_{\left(k_{j}+1\right) T}\right)\right]+o\left(\epsilon^{N}\right) \\
& =\sum_{n=0}^{N} \epsilon^{n} \sum_{\vec{k}_{\delta}}^{(n)} \frac{1}{\delta !} \mathbf{E}\left[\Phi^{(\delta)}\left(g_{1 T}\right) X^{\vec{k}_{\delta}}\right]+o\left(\epsilon^{N}\right) \\
& =\sum_{n=0}^{N} \epsilon^{n} \sum_{\vec{k}_{\delta}}^{(n)} \frac{1}{\delta !} \int_{\mathbf{R}} \Phi^{(\delta)}(x) \mathbf{E}\left[X^{\vec{k}_{\delta}} \mid g_{1 T}=x\right] f_{g_{1 T}}(x) d x+o\left(\epsilon^{N}\right) \\
& =\sum_{n=0}^{N} \epsilon^{n} \sum_{\vec{k}_{\delta}}^{(n)} \frac{1}{\delta !} \int_{\mathbf{R}} \Phi(x)(-1)^{\delta} \frac{d^{\delta}}{d x^{\delta}}\left\{\mathbf{E}\left[X^{\vec{k}_{\delta}} \mid g_{1 T}=x\right] f_{g_{1 T}}(x)\right\} d x+o\left(\epsilon^{N}\right)
\end{aligned}
$$

where $\Phi^{(\delta)}\left(g_{1 T}\right)=\left.\frac{\partial^{\delta} \Phi(x)}{\partial x^{\delta}}\right|_{x=g_{1 T}}$,

$$
X^{\vec{k}_{\delta}}=\prod_{j=1}^{\delta} g_{\left(k_{j}+1\right) T}
$$

for $\vec{k}_{\delta} \in L_{n, \delta}$, and

$$
\sum_{\vec{k}_{\delta}}^{(n)}=\sum_{\delta=1}^{n} \sum_{\vec{k}_{\delta} \in L_{n, \delta}}
$$

In the preceding works on application of the asymptotic expansion, conditional expectations in (7) were directly computed with some formulas given in [21] or [23] (for example, see Appendix B of [23]). Recently, while the formulas had been given up to the third order by those papers, [26] developed a high-order computation scheme for the conditional expectations using the fact that each of these $\left\{A_{k, t}^{j}\right\}_{j, k},\left\{g_{n T}\right\}_{n}$ and also $\left\{X^{\vec{k}_{\delta}}\right\}_{\vec{k}_{\delta}}$ can be decomposed into a finite sum of iterated multiple Wiener-Itô integrals by Itô's formula, and a certain property of iterated multiple Wiener-Itô integrals (see Nualart, Üstünel and Zakai [17] and Section 4 of [26]). On the other hand, as shown in the next section, this paper develops a new method computing unconditional expectations instead of the conditional ones.

\section{A New Computational Scheme}

In this section we propose the new computational scheme in the asymptotic expansion, which is an alternative to the direct calculation method for the conditional expectations given by [26].

To compute the conditional expectations in the right hand side of (7), we use the following lemma which can be derived from a property of Hermite polynomials and leads us to compute the unconditional expectations instead of the conditional ones.

Lemma 1 Let $(\Omega, F, P)$ be a probability space. Suppose that $X \in L^{2}(\Omega, P)$ and $Z$ is a random variable with Gaussian distribution with mean 0 and variance $\Sigma$. 
Then, the conditional expectation $E[X \mid Z=x]$ has the following expansion in $L^{2}(\mathbf{R}, \mu)$ where $\mu$ is the Gaussian measure on $\mathbf{R}$ with mean 0 and variance $\Sigma$ :

$$
E[X \mid Z=x]=\sum_{n=0}^{\infty} \frac{a_{n}}{\Sigma^{n}} H_{n}(x ; \Sigma)
$$

where $H_{n}(x ; \Sigma)$ is the Hermite polynomial of degree $n$ which is defined as

$$
H_{n}(x ; \Sigma)=(-\Sigma)^{n} e^{x^{2} / 2 \Sigma} \frac{d^{n}}{d x^{n}} e^{-x^{2} / 2 \Sigma}
$$

and coefficients $a_{n}$ are given by

$$
a_{n}=\left.\frac{1}{n !} \frac{1}{i^{n}} \frac{\partial^{n}}{\partial \xi^{n}}\right|_{\xi=0}\left\{e^{\frac{\xi^{2}}{2} \Sigma} \mathbf{E}\left[e^{i \xi Z} X\right]\right\} .
$$

(proof) See Section 5.1.

Here, we define $\hat{g}_{1}=\left\{\hat{g}_{1 t} ; t \in \mathbf{R}^{+}\right\}$and $Z^{\langle\xi\rangle}=\left\{Z_{t}^{\langle\xi\rangle} ; t \in \mathbf{R}^{+}\right\}$as the stochastic processes

$$
\hat{g}_{1 t}=\int_{0}^{t} \hat{V}\left(X_{u}^{(0)}, u\right) d W_{u}
$$

and

$$
Z_{t}^{\langle\xi\rangle}=\exp \left(i \xi \hat{g}_{1 t}+\frac{\xi^{2}}{2} \Sigma_{t}\right)
$$

respectively.

Then, from Lemma 1, the conditional expectations appearing in the right hand side of the equation (7) is expressed as

$$
\begin{aligned}
\mathbf{E}\left[X^{\vec{k}_{\delta}} \mid g_{1 T}=x\right] & =\mathbf{E}\left[X^{\vec{k}_{\delta}} \mid \hat{g}_{1 T}=x-C\right] \\
& =\sum_{l=0}^{\infty} \frac{a_{l}^{\vec{k}_{\delta}}}{\Sigma_{T}^{l}} H_{l}\left(x-C, \Sigma_{T}\right)
\end{aligned}
$$

where

$$
a_{l}^{\vec{k}_{\delta}}=\left.\frac{1}{l !} \frac{1}{i^{l}} \frac{\partial^{l}}{\partial \xi^{l}} \mathbf{E}\left[X^{\vec{k}_{\delta}} Z_{T}^{\langle\xi\rangle}\right]\right|_{\xi=0} .
$$

Here it is noted that with this expression we now need to compute unconditional expectations $\mathbf{E}\left[X^{\vec{k}_{\delta}} Z_{T}^{\langle\xi\rangle}\right]$ instead of the conditional expectations.

\subsection{The Asymptotic Expansion of Density Function}

In this subsection, we explain the new computational method through deriving a general formula for the expansion (7) with an arbitrary specification of its order $N$. In particular, we show that the coefficients in the expansion are obtained through a system of ordinary differential equations that is solved easily.

First, we define $\eta_{\vec{l}_{\beta}}^{\vec{d}_{\beta}}(t ; \xi)$ for $\vec{l}_{\beta} \in L_{n, \beta}$ and $\vec{d}_{\beta} \in\{1, \cdots, d\}^{\beta}(n \geq \beta \geq 1)$ as

$$
\eta_{\vec{l}_{\beta}}^{\vec{d}_{\beta}}(t ; \xi)=\mathbf{E}\left[\left(\prod_{j=1}^{\beta} A_{l_{j} t}^{d_{j}}\right) Z_{t}^{\langle\xi\rangle}\right],
$$

and for $n=0$ as

$$
\eta_{(\emptyset)}^{(\emptyset)}(t ; \xi)=\mathbf{E}\left[Z_{t}^{\langle\xi\rangle}\right]
$$


Then, unconditional expectations $\mathbf{E}\left[X^{\vec{k}_{\delta}} Z_{T}^{\langle\xi\rangle}\right]$ appearing in the definition of $a_{l}^{\vec{k}_{\delta}}(11)$ can be written in terms of $\eta$ as follows:

$$
\begin{aligned}
\mathbf{E}\left[X^{\vec{k}_{\delta}} Z_{T}^{\langle\xi\rangle}\right] & =\mathbf{E}\left[\left(\prod_{j=1}^{\delta} g_{\left(k_{j}+1\right) T}\right) Z_{T}^{\langle\xi\rangle}\right] \\
& =\mathbf{E}\left[\left(\prod_{j=1}^{\delta} \sum_{\vec{l}_{\beta_{j}}^{j}, \vec{d}_{\beta_{j}}^{j}}^{\left(k_{j}+1\right)} \frac{1}{\beta_{j} !} \partial_{\vec{d}_{\beta_{j}}^{j}}^{\beta_{j}} g\left(X_{T}^{(0)}\right) A_{l_{1}^{j} T}^{d_{1}^{j}} \cdots A_{l_{\beta_{j}}^{j}}^{d_{\beta_{j}}^{j}}\right) Z_{T}^{\langle\xi\rangle}\right] \\
& =\sum_{\vec{l}_{\beta_{1}}^{1}, \vec{d}_{\beta_{1}}^{1}}^{\left(k_{1}+1\right)} \sum_{\vec{l}_{\beta_{\delta}}^{\delta}, \vec{d}_{\beta_{\delta}}^{\delta}}^{\left(k_{\delta}+1\right)}\left(\prod_{j=1}^{\delta} \frac{1}{\beta_{j} !} \partial_{\vec{d}_{\beta_{j}}^{j}}^{\beta_{j}} g\left(X_{T}^{(0)}\right)\right) \eta_{\vec{l}_{\beta_{1}}^{1} \otimes \cdots \otimes \vec{l}_{\beta_{\delta}}^{\delta}}^{\vec{d}_{\delta}}(T ; \xi)
\end{aligned}
$$

where

$$
\begin{aligned}
\vec{d}_{\beta_{i}}^{\imath} \otimes \vec{d}_{\beta_{j}}^{j} & :=\left(d_{1}^{i}, \cdots, d_{\beta_{i}}^{i}, d_{1}^{j}, \cdots, d_{\beta_{j}}^{j}\right), \\
\vec{l}_{\beta_{i}}^{i} \otimes \vec{l}_{\beta_{j}}^{j} & :=\left(l_{1}^{i}, \cdots, l_{\beta_{i}}^{i}, l_{1}^{j}, \cdots, l_{\beta_{j}}^{j}\right) .
\end{aligned}
$$

So, we have to calculate $\eta_{\vec{l}_{\beta}} \vec{d}_{\beta}(T ; \xi)$ to evaluate the asymptotic expansion (7).

In the following, we derive a system of ODEs satisfied by these $\left\{\eta_{\vec{l}_{\beta}}^{\vec{d}_{\beta}}\right\}$. Before showing a general result, we first derive the ODEs for few leading-low-order terms explicitly to give a better intuition of a key idea of our method. Consider the evaluation of $\eta_{(2)}^{j}(T ; \xi)=E\left[A_{2 T}^{j} Z_{T}^{\langle\xi\rangle}\right]$ which appears in the $\epsilon$-order. Here, for simplicity, we assume that $V_{0}$ does not depend on $\epsilon$, and write $V_{0}(x, \epsilon)$ as $V_{0}(x)$. First, applying Itô's formula to $A_{2 t}^{j} Z_{t}^{\langle\xi\rangle}$, we have

$$
\begin{aligned}
d\left(A_{2 t}^{j} Z_{t}^{\langle\xi\rangle}\right)= & A_{2 t}^{j} d Z_{t}^{\langle\xi\rangle}+Z_{t}^{\langle\xi\rangle} d A_{2 t}^{j}+d\left\langle A_{2}^{j}, Z^{\langle\xi\rangle}\right\rangle_{t} \\
= & \left\{(i \xi) \sum_{j^{\prime}=1}^{d} A_{1 t}^{j^{\prime}} Z_{t}^{\langle\xi\rangle} \hat{V}\left(X_{t}^{(0)}, t\right) \partial_{j^{\prime}} V^{j}\left(X_{t}^{(0)}\right)^{\prime}+\sum_{j^{\prime}=1}^{d} A_{2 t}^{j^{\prime}} Z_{t}^{\langle\xi\rangle} \partial_{j^{\prime}} V_{0}^{j}\left(X_{t}^{(0)}\right)\right. \\
& \left.+\frac{1}{2} \sum_{j^{\prime}, k^{\prime}=1}^{d} A_{1 t}^{j^{\prime}} A_{1 t}^{k^{\prime}} Z_{t}^{\langle\xi\rangle} \partial_{j^{\prime}} \partial_{k^{\prime}} V_{0}^{j}\left(X_{t}^{(0)}\right)\right\} d t \\
& +\left\{(i \xi) A_{2 t}^{j} Z_{t}^{\langle\xi\rangle} \hat{V}\left(X_{t}^{(0)}, t\right)+\sum_{j^{\prime}=1}^{d} A_{1 t}^{j^{\prime}} Z_{t}^{\langle\xi\rangle} \partial_{j^{\prime}} V^{j}\left(X_{t}^{(0)}\right)\right\} d W_{t} .
\end{aligned}
$$

Since the last term is a martingale, taking expectation on both sides, we have the following ordinary differential equation for $\eta_{(2)}^{j}$ :

$$
\begin{aligned}
\frac{d}{d t} \eta_{(2)}^{j}(t ; \xi)= & (i \xi) \sum_{j^{\prime}=1}^{d} \eta_{(1)}^{j^{\prime}}(t ; \xi) \hat{V}\left(X_{t}^{(0)}, t\right) \partial_{j^{\prime}} V^{j}\left(X_{t}^{(0)}\right)^{\prime} \\
& +\sum_{j^{\prime}=1}^{d} \eta_{(2)}^{j^{\prime}}(t ; \xi) \partial_{j^{\prime}} V_{0}^{j}\left(X_{t}^{(0)}\right)+\frac{1}{2} \sum_{j^{\prime}, k^{\prime}=1}^{d} \eta_{(1,1)}^{j^{\prime}, k^{\prime}}(t ; \xi) \partial_{j^{\prime}} \partial_{k^{\prime}} V_{0}^{j}\left(X_{t}^{(0)}\right) .
\end{aligned}
$$

Here, $\eta_{(1)}^{j}(j=1, \cdots, d)$ appearing in the right hand side of the above ODE are 
evaluated in the similar manner:

$$
\begin{aligned}
d\left(A_{1 t}^{j} Z_{t}^{\langle\xi\rangle}\right)= & A_{1 t}^{j} d Z_{t}^{\langle\xi\rangle}+Z_{t}^{\langle\xi\rangle} d A_{1 t}^{j}+d\left\langle A_{1}^{j}, Z^{\langle\xi\rangle}\right\rangle_{t} \\
= & \left\{(i \xi) Z_{t}^{\langle\xi\rangle} \hat{V}\left(X_{t}^{(0)}, t\right) V^{j}\left(X_{t}^{(0)}\right)^{\prime}+\sum_{j^{\prime}=1}^{d} A_{1 t}^{j^{\prime}} Z_{t}^{\langle\xi\rangle} \partial_{j^{\prime}} V_{0}^{j}\left(X_{t}^{(0)}\right)\right\} d t \\
& +\left\{(i \xi) A_{1 t}^{j} Z_{t}^{\langle\xi\rangle} \hat{V}\left(X_{t}^{(0)}, t\right)+Z_{t}^{\langle\xi\rangle} V^{j}\left(X_{t}^{(0)}\right)\right\} d W_{t},
\end{aligned}
$$

hence, we have

$$
\frac{d}{d t} \eta_{(1)}^{j}(t ; \xi)=(i \xi) \hat{V}\left(X_{t}^{(0)}, t\right) V^{j}\left(X_{t}^{(0)}\right)^{\prime}+\sum_{j^{\prime}=1}^{d} \eta_{(1)}^{j^{\prime}}(t ; \xi) \partial_{j^{\prime}} V_{0}^{j}\left(X_{t}^{(0)}\right) .
$$

$\eta_{(1,1)}^{j, k}$ and other higher-order terms can be evaluated in the same way. The key observation is that each ODE does not involve any higher-order terms, and only lower- or the same order- terms appear in the right hand side of the ODE. So, one can easily solve (analytically or numerically) the system of ODEs and evaluate the expectations.

The following theorem provides a way to calculate general $\eta_{\vec{l}_{\beta}}^{\vec{d}_{\beta}}(T ; \xi)$ as a solution to the system of the ordinary differential equations:

Theorem 1 For $\eta_{\vec{l}_{\beta}}^{\vec{d}_{\beta}}(t ; \xi)$ defined in (12), the following system of ordinary differential equations is satisfied:

$$
\begin{aligned}
\frac{d}{d t}\left\{\eta_{\vec{l}_{\beta}}^{\vec{d}_{\beta}}(t ; \xi)\right\} & =\sum_{k=1}^{\beta} \frac{1}{l_{k} !}\left\{\eta_{\vec{l}_{\beta / k}}^{\vec{d}_{\beta / k}}(t ; \xi)\right\}\left\{\partial_{\epsilon}^{l_{k}} V_{0}^{d_{k}}\left(X_{t}^{(0)}, 0\right)\right\} \\
& +\sum_{k=1}^{\beta} \sum_{l=1}^{l_{k}} \sum_{\vec{m}_{\gamma}, \vec{d}_{\gamma}}^{(l)} \frac{1}{\left(l_{k}-l\right) !} \frac{1}{\gamma !}\left\{\eta_{\left(\vec{l}_{\beta / k}\right) \otimes \vec{m}_{\gamma}}^{\left(\vec{d}_{\beta / k}\right) \otimes \vec{d}_{\gamma}}(t ; \xi)\right\}\left\{\partial_{\vec{d}_{\gamma}}^{\gamma} \partial_{\epsilon}^{l_{k}-l} V_{0}^{d_{k}}\left(X_{t}^{(0)}, 0\right)\right\} \\
& +\sum_{k, m=1}^{\beta} \sum_{k<m}^{\left(l_{k}-1\right)} \sum_{\vec{m}_{\gamma}, \overrightarrow{\vec{d}}_{\gamma}}^{\left(l_{m}-1\right)} \frac{1}{\gamma ! \delta !}\left\{\eta_{\vec{m}_{\delta}, \vec{d}_{\delta}}^{\left.\left(\vec{d}_{\beta / k, m}\right) \otimes \vec{d}_{\gamma / k} \otimes \vec{d}_{\delta}\right) \otimes \vec{m}_{\gamma} \otimes \vec{m}_{\delta}}(t ; \xi)\right\}\left\{\partial_{\vec{d}_{\gamma}^{\gamma}}^{\gamma} V^{d_{k}}\left(X_{t}^{(0)}\right)\right\}\left\{\partial_{\vec{d}_{\delta}}^{\delta} V^{d_{m}}\left(X_{t}^{(0)}\right)\right\} \\
& +(i \xi) \sum_{k=1}^{\beta} \sum_{\vec{m}_{\gamma}, \vec{d}_{\gamma}}^{\left(l_{k}-1\right)} \frac{1}{\gamma !}\left\{\eta_{\left(\vec{l}_{\beta / k}\right) \otimes \vec{m}_{\gamma}}^{\left(\vec{d}_{\beta / n}\right) \otimes \vec{d}_{\gamma}}(t ; \xi)\right\}\left\{\partial_{\vec{d}_{\gamma}}^{\gamma} V^{d_{k}}\left(X_{t}^{(0)}\right)\right\} \hat{V}\left(X_{t}^{(0)}, t\right)
\end{aligned}
$$

where

$$
\begin{aligned}
\vec{l}_{\beta / k} & :=\left(l_{1}, \cdots, l_{k-1}, l_{k+1}, \cdots, l_{\beta}\right) \\
\vec{l}_{\beta / k, n} & :=\left(l_{1}, \cdots, l_{k-1}, l_{k+1}, \cdots, l_{n-1}, l_{n+1}, \cdots, l_{\beta}\right), 1 \leq k<n \leq \beta \\
\vec{l}_{\beta} \otimes \vec{m}_{\gamma} & :=\left(l_{1}, \cdots, l_{\beta}, m_{1}, \cdots, m_{\gamma}\right)
\end{aligned}
$$

for $\vec{l}_{\beta}=\left(l_{1}, \cdots, l_{\beta}\right)$ and $\vec{m}_{\gamma}=\left(m_{1}, \cdots, m_{\gamma}\right)$.

(Proof) See Section 5.2.

Remark 1 Due to the hierarchical structure of the ODEs with respect to $n=$ $\sum_{j=1}^{\beta} l_{j}$ and $\eta_{(\emptyset)}^{(\emptyset)}(t ; \xi)=\mathbf{E}\left[Z_{t}^{\langle\xi\rangle}\right]=1$, one can easily solve these ODEs successively from lower-order terms to higher-order terms with initial conditions $\eta_{\vec{l}_{\beta}}^{\vec{d}_{\beta}}(0 ; \xi)=0$ for $\left(\vec{l}_{\beta}, \vec{d}_{\beta}\right) \neq(\emptyset, \emptyset)$. 
Remark 2 Further, due to the structure of the system of the differential equations, it is easily shown by induction that each $\eta_{\vec{l}_{\beta}}^{\vec{d}_{\beta}}(t ; \xi)$ is expressed as a polynomial of degree $n=\sum_{j=1}^{\beta} l_{j}$ with respect to $(i \xi)$. Then, we can also show that $\mathbf{E}\left[X^{\vec{k}_{\delta}} Z_{T}^{\langle\xi\rangle}\right]$ is a polynomial of degree $(n+\delta)$ with respect to $(i \xi)$, and thus $a_{l}^{\vec{k}_{\delta}}=0(l>n+\delta)$ for $\vec{k}_{\delta} \in L_{n, \delta}$. This ensures a convergence of the infinite sum in (10).

Then, from Lemma 1 and (7), we have the following expression of $\mathbf{E}\left[\Phi\left(G^{(\epsilon)}\right)\right]$ :

$$
\begin{aligned}
\mathbf{E}\left[\Phi\left(G^{(\epsilon)}\right)\right] & =\sum_{n=0}^{N} \epsilon^{n} \sum_{\vec{k}_{\delta}}^{(n)} \frac{1}{\delta !} \int_{\mathbf{R}} \Phi(x)(-1)^{\delta} \frac{d^{\delta}}{d x^{\delta}}\left\{\sum_{l=0}^{n+\delta} \frac{a_{l}^{\vec{k}_{\delta}}}{\Sigma_{T}^{l}} H_{l}\left(x-C, \Sigma_{T}\right) f_{g_{1 T}}(x)\right\} d x+o\left(\epsilon^{N}\right) \\
& =\sum_{n=0}^{N} \epsilon^{n} \sum_{\vec{k}_{\delta}}^{(n)} \frac{1}{\delta !} \int_{\mathbf{R}} \Phi(x)\left\{\sum_{l=0}^{n+\delta} \frac{a_{l}^{\vec{k}_{\delta}}}{\Sigma_{T}^{l+\delta}} H_{l+\delta}\left(x-C, \Sigma_{T}\right) f_{g_{1 T}}(x)\right\} d x+o\left(\epsilon^{N}\right)
\end{aligned}
$$

Here we used the relation

$$
\frac{d^{\delta}}{d x^{\delta}}\left\{H_{l}\left(x-C, \Sigma_{T}\right) f_{g_{1 T}}(x)\right\}=(-1)^{\delta} \frac{1}{\Sigma_{T}^{\delta}} H_{l+\delta}\left(x-C, \Sigma_{T}\right) f_{g_{1 T}}(x) .
$$

In particular, let $\Phi$ be the delta function at $x \in \mathbf{R}, \delta_{x}$, we obtain the asymptotic expansion of the density of $G^{(\epsilon)}$ :

$$
\begin{aligned}
f_{G^{(\epsilon)}}(x) & =\mathbf{E}\left[\delta_{x}\left(G^{(\epsilon)}\right)\right] \\
& =\sum_{n=0}^{N} \epsilon^{n} \sum_{\vec{k}_{\delta}}^{(n)} \frac{1}{\delta !} \sum_{l=0}^{n+\delta} \frac{a_{l}^{\vec{k}_{\delta}}}{\Sigma_{T}^{l+\delta}} H_{l+\delta}\left(x-C, \Sigma_{T}\right) f_{g_{1 T}}(x)+o\left(\epsilon^{N}\right) .
\end{aligned}
$$

We summarize the discussion above as the following theorem:

Theorem 2 The asymptotic expansion of the density function of $G^{(\epsilon)}$ up to $\epsilon^{N}$-order is given by

$$
f_{G^{(\epsilon)}}(x)=f_{g_{1 T}}(x)+\sum_{n=1}^{N} \epsilon^{n}\left(\sum_{m=0}^{3 n} C_{n m} H_{m}\left(x-C, \Sigma_{T}\right)\right) f_{g_{1 T}}(x)+o\left(\epsilon^{N}\right)
$$

where

$$
\begin{aligned}
& C_{n m}=\frac{1}{\Sigma_{T}^{m}} \sum_{\delta=1}^{m} \sum_{\vec{k}_{\delta} \in L_{n, \delta}} \sum_{\vec{l}_{\beta_{1}}^{1}, \vec{d}_{\beta_{1}}^{1}}^{\left(k_{1}+1\right)} \cdots \sum_{\vec{l}_{\beta_{\delta}}^{\vec{d}}{\overrightarrow{d_{\beta}}}_{\beta_{\delta}}^{\vec{b}}}^{\left(k_{\delta}+1\right)} \frac{1}{\delta !(m-\delta) !} \\
& \left.\left(\prod_{j=1}^{\delta} \frac{1}{\beta_{j} !} \partial_{\vec{d}_{\beta_{j}}^{j}}^{\beta_{j}} g\left(X_{T}^{(0)}\right)\right) \frac{1}{i^{m-\delta}} \frac{\partial^{m-\delta}}{\partial \xi^{m-\delta}}\left\{\eta_{\vec{l}_{\beta_{1}}^{1} \otimes \cdots \otimes \otimes \vec{l}_{\beta_{\delta}}^{\delta}}^{\vec{d}_{\beta_{\delta}} \otimes \cdots \otimes \vec{d}_{\delta}^{\delta}}(T ; \xi)\right\}\right|_{\xi=0}
\end{aligned}
$$

and $\eta_{\vec{l}_{\beta}}{\overrightarrow{\vec{B}_{\beta}}}_{\boldsymbol{1}}(T ; \xi)$ are obtained as a solution to the system of ODEs given in Theorem 1. 


\subsection{Asymptotic Expansion of Option Prices}

We apply the asymptotic expansion to option pricing. We consider a plain vanilla option on the underlying asset $g\left(X_{T}^{(\epsilon)}\right)$ whose dynamics is given by (1).

For example, an asymptotic expansion up to $\epsilon^{(N+1)}$ of a call option price at time 0 with maturity $T$ and strike price $K$ where $K=X_{T}^{(0)}-\epsilon y$ for arbitrary $y \in \mathbf{R}$ is given by

$$
\begin{aligned}
C(K, T) & =P(0, T) \mathbf{E}\left[\max \left(g\left(X_{T}^{(\epsilon)}\right)-K, 0\right)\right] \\
& =\epsilon P(0, T) \int_{-y}^{\infty}(x+y) f_{G^{(\epsilon)}, N}(x) d x+o\left(\epsilon^{(N+1)}\right) .
\end{aligned}
$$

Here, $P(0, T)$ denotes the price at time 0 of a zero coupon bond with maturity $T$ and $f_{G^{(\epsilon)}, N}$ is the asymptotic expansion of the density of $G^{(\epsilon)}$ up to $\epsilon^{N}$-order given by (17):

$$
f_{G^{(\epsilon)}, N}(x)=f_{g_{1 T}}(x)+\sum_{n=1}^{N} \epsilon^{n}\left(\sum_{m=0}^{3 n} C_{n m} H_{m}\left(x-C, \Sigma_{T}\right)\right) f_{g_{1 T}}(x)
$$

Integrals appearing in the right hand side of (19) can be calculated by following formulas related to the Hermite polynomials

$$
\begin{aligned}
\int_{-y}^{\infty} H_{k}(x ; \Sigma) f_{g_{1 T}}(x) d x= & \Sigma H_{k-1}(-y ; \Sigma) f_{g_{1 T}}(y)(k \geq 1), \\
\int_{-y}^{\infty} x H_{k}(x ; \Sigma) f_{g_{1 T}}(x) d x= & -\Sigma y H_{k-1}(-y ; \Sigma) f_{g_{1 T}}(y) \\
& +\Sigma^{2} H_{k-2}(-y ; \Sigma) f_{g_{1 T}}(y)(k \geq 2) .
\end{aligned}
$$

\subsection{A Log-Normal Asymptotic Expansion}

In this subsection, we develop a slightly different expansion from the one introduced in the previous subsection.

Suppose that an underlying one-dimensional asset process $S^{(\epsilon)}$ and $d$-dimensional stochastic process $X^{(\epsilon)}$ follow

$$
\begin{aligned}
d S_{t}^{(\epsilon)} & =g\left(X_{t}^{(\epsilon)}\right) S_{t}^{(\epsilon)} \bar{\sigma} d W_{t} ; \quad S_{0}^{(\epsilon)}=s_{0}, \\
d X_{t}^{(\epsilon)} & =V_{0}\left(X_{t}^{(\epsilon)}, \epsilon\right) d t+\epsilon V\left(X_{t}^{(\epsilon)}\right) d W_{t} ; \quad X_{0}^{(\epsilon)}=x_{0} \in \mathbf{R}^{d}
\end{aligned}
$$

respectively, where $g: \mathbf{R}^{d} \rightarrow \mathbf{R}$ and $\bar{\sigma}$ is a constant vector in $\mathbf{R}^{r}$. First, let we define $\hat{X}^{(\epsilon)}$ as

$$
\hat{X}_{t}^{(\epsilon)}=\log \left(\frac{S_{t}^{(\epsilon)}}{s_{0}}\right)
$$

Then, we have

$$
\hat{X}_{t}^{(\epsilon)}=-\frac{|\bar{\sigma}|^{2}}{2} \int_{0}^{t} g\left(X_{u}^{(\epsilon)}\right)^{2} d u+\int_{0}^{t} g\left(X_{u}^{(\epsilon)}\right) \bar{\sigma} d W_{u},
$$

and note that

$$
\hat{X}_{T}^{(0)} \sim N\left(\hat{\mu}_{T}, \hat{\Sigma}_{T}\right)
$$

where

$$
\begin{aligned}
& \hat{\mu}_{T}=-\frac{|\bar{\sigma}|^{2}}{2} \int_{0}^{T} g\left(X_{u}^{(0)}\right)^{2} d u=-\frac{1}{2} \hat{\Sigma}_{T}, \\
& \hat{\Sigma}_{T}=|\bar{\sigma}|^{2} \int_{0}^{T} g\left(X_{u}^{(0)}\right)^{2} d u .
\end{aligned}
$$


Moreover, an asymptotic expansion of $\hat{X}_{T}^{(\epsilon)}$ up to $\epsilon^{N}$-order is expressed as

$$
\hat{X}_{T}^{(\epsilon)}=\hat{X}_{T}^{(0)}+\sum_{n=1}^{N} \epsilon^{n} \hat{A}_{n T}+o\left(\epsilon^{N}\right),
$$

where $\hat{A}_{n t}=\left.\frac{1}{n !} \frac{\partial^{n} \hat{X}_{t}^{(\epsilon)}}{\partial \epsilon^{n}}\right|_{\epsilon=0}$. Note that $S_{T}^{(\epsilon)}$ is now expanded around a log-normal distribution since $\hat{X}_{T}^{(0)}$ has the Gaussian distribution (hereafter we call this expansion 'the log-normal asymptotic expansion' of $S_{T}^{(\epsilon)}$ in contrast to calling the expansion in the previous subsection 'the normal asymptotic expansion').

Next, define $Z^{\langle\xi\rangle}=\left\{Z_{t}^{\langle\xi\rangle} ; t \in \mathbf{R}^{+}\right\}$as

$$
Z_{t}^{\langle\xi\rangle}=\exp \left(i \xi \int_{0}^{t} g\left(X_{u}^{(0)}\right) \bar{\sigma} d W_{u}\right)
$$

Then, the result in the previous subsection is applied to deriving the density function of $\hat{X}_{T}^{(\epsilon)}$ with replacement of $G^{(\epsilon)}$ by $\hat{X}_{T}^{(\epsilon)}$.

Similar to the normal case, the log-normal asymptotic expansion of the price of the call option on $\hat{X}_{T}^{(\epsilon)}$ is given by

$$
C(K, T)=P(0, T) \int_{\log \frac{K}{s_{0}}}^{\infty}\left(s_{0} e^{x}-K\right) f_{\hat{X}_{T}^{(\epsilon)}}(x) d x .
$$

We also remark that as shown in Takahashi and Yamada [29], the asymptotic expansion approach can be applied to a shifted log-normal model, sometimes called a displaced diffusion model (Rubinstein [18]), under stochastic volatility environments. Hence, the method for high-order expansions introduced in this paper can be applied to the model. More specifically, a stochastic volatility version of this model in the asymptotic expansion framework is expressed as follows:

$$
\begin{aligned}
d S_{t}^{(\epsilon)} & =g\left(X_{t}^{(\epsilon)}\right)\left(S_{t}^{(\epsilon)}+\alpha\right) \bar{\sigma} d W_{t} ; \quad S_{0}^{(\epsilon)}=s_{0} \\
d X_{t}^{(\epsilon)} & =V_{0}\left(X_{t}^{(\epsilon)}, \epsilon\right) d t+\epsilon V\left(X_{t}^{(\epsilon)}\right) d W_{t} ; \quad X_{0}^{(\epsilon)}=x_{0} \in \mathbf{R}^{d}
\end{aligned}
$$

where $\alpha$ is a constant. Once we define $\hat{S}_{t}^{(\epsilon)}:=S_{t}^{(\epsilon)}+\alpha$, the same method as above is easily applied since the model is now described as

$$
\begin{aligned}
d \hat{S}_{t}^{(\epsilon)} & =g\left(X_{t}^{(\epsilon)}\right) \hat{S}_{t}^{(\epsilon)} \bar{\sigma} d W_{t} ; \quad \hat{S}_{0}^{(\epsilon)}=s_{0}+\alpha \\
d X_{t}^{(\epsilon)} & =V_{0}\left(X_{t}^{(\epsilon)}, \epsilon\right) d t+\epsilon V\left(X_{t}^{(\epsilon)}\right) d W_{t} ; \quad X_{0}^{(\epsilon)}=x_{0} \in \mathbf{R}^{d}
\end{aligned}
$$

and the payoffs of call and put options with strike $K$ and maturity $T$ are expressed as $\left(\hat{S}_{T}^{(\epsilon)}-(K+\alpha)\right)^{+}$and $\left((K+\alpha)-\hat{S}_{T}^{(\epsilon)}\right)^{+}$respectively.

\section{Numerical Examples}

\section{1 $\lambda$-SABR model}

In this section, we test effectiveness of the asymptotic expansion method described in the previous section through numerical examples. Also, we compare approximation accuracy of our method with that of another existing approximation method. 


\subsubsection{Asymptotic Expansion of the $\lambda$-SABR Model}

To test efficiency of the expansion, we first consider a European plain-vanilla call and put prices under the following $\lambda$-SABR model [12] (interest rate $=0 \%$ ) :

$$
\begin{aligned}
d S^{(\epsilon)}(t) & =\epsilon \sigma^{(\epsilon)}(t)\left(S^{(\epsilon)}(t)\right)^{\beta} d W_{t}^{1}, \\
d \sigma^{(\epsilon)}(t) & =\lambda\left(\theta-\sigma^{(\epsilon)}(t)\right) d t+\epsilon \nu_{1} \sigma^{(\epsilon)}(t) d W_{t}^{1}+\epsilon \nu_{2} \sigma^{(\epsilon)}(t) d W_{t}^{2}, \\
S^{(\epsilon)}(0) & =S_{0}, \quad \sigma^{(\epsilon)}(0)=\sigma_{0},
\end{aligned}
$$

where $\nu_{1}=\rho \nu, \nu_{2}=\left(\sqrt{1-\rho^{2}}\right) \nu$ (an instantaneous correlation between $S^{(\epsilon)}$ and $\sigma^{(\epsilon)}$ is $\left.\rho \in[-1,1]\right)$. Note that when $\lambda=0$ the model becomes the original SABR model [7]. Rigorously speaking, this model does not satisfy the regularity conditions stated in pp.3-4 of Section 2 since the coefficient function $V^{1}(\sigma, s)=$ $\sigma s^{\beta}$ is unbounded and has non-smooth derivatives at $s=0$. However, as seen in the following, our method is (formally) applicable to this model and gives better accuracies for approximate prices in higher-order expansions for various ranges of strikes and parameters.

To compute an option price on $S^{(\epsilon)}$, we need the density function of $S_{T}^{(\epsilon)}$ whose asymptotic expansion is given by (17) with setting $g(S, \sigma)=S$. The asymptotic expansion of the density function is obtained by solving the system of the ordinary differential equations given in Theorem 1. For example, the corresponding differential equations up to the second order are given by

$$
\begin{aligned}
\frac{d}{d t} \eta_{(1)}^{S}(t ; \xi) & =(i \xi)\left(S_{t}^{(0)}\right)^{2 \beta}\left(\sigma_{t}^{(0)}\right)^{2}, \\
\frac{d}{d t} \eta_{(1)}^{\sigma}(t ; \xi) & =(i \xi) \nu_{1}\left(S_{t}^{(0)}\right)^{\beta}\left(\sigma_{t}^{(0)}\right)^{2}-\lambda \eta_{(1)}^{\sigma}(t ; \xi), \\
\frac{d}{d t} \eta_{(2)}^{S}(t ; \xi) & =(i \xi) \beta\left(S_{t}^{(0)}\right)^{2 \beta-1}\left(\sigma_{t}^{(0)}\right)^{2} \eta_{(1)}^{S}(t ; \xi)+(i \xi)\left(S_{t}^{(0)}\right)^{2 \beta} \sigma_{t}^{(0)} \eta_{(1)}^{\sigma}(t ; \xi),
\end{aligned}
$$

where $S_{t}^{(0)}=S_{0}$ and $\sigma_{t}^{(0)}=e^{-\lambda t}\left(\sigma_{0}-\theta\right)+\theta$. Since these equations are linear and have the hierarchical structure, one can easily integrate them as

$$
\begin{aligned}
\eta_{(1)}^{S}(t ; \xi)= & (i \xi) \int_{0}^{t}\left(S_{t_{1}}^{(0)}\right)^{2 \beta}\left(\sigma_{t_{1}}^{(0)}\right)^{2} d t_{1} \\
\eta_{(1)}^{\sigma}(t ; \xi)= & (i \xi) \int_{0}^{t} e^{-\lambda\left(t-t_{1}\right)} \nu_{1}\left(S_{t_{1}}^{(0)}\right)^{\beta}\left(\sigma_{t_{1}}^{(0)}\right)^{2} d t_{1} \\
\eta_{(2)}^{S}(t ; \xi)= & (i \xi)^{2} \int_{0}^{t} \int_{0}^{t_{1}} \beta\left(S_{t_{1}}^{(0)}\right)^{2 \beta-1}\left(\sigma_{t_{1}}^{(0)}\right)^{2}\left(S_{t_{2}}^{(0)}\right)^{2 \beta}\left(\sigma_{t_{2}}^{(0)}\right)^{2} d t_{2} d t_{1} \\
& +(i \xi)^{2} \int_{0}^{t} \int_{0}^{t_{1}} e^{-\lambda\left(t_{1}-t_{2}\right)}\left(S_{t_{1}}^{(0)}\right)^{2 \beta} \sigma_{t_{1}}^{(0)} \nu_{1}\left(S_{t_{2}}^{(0)}\right)^{\beta}\left(\sigma_{t_{2}}^{(0)}\right)^{2} d t_{2} d t_{1}
\end{aligned}
$$

Integrals appearing in the right hand side are analytically evaluated, which is omitted due to the limitation of the space (they are available upon request).

Then, from Theorem 2 the asymptotic expansion of the density function of $G^{(\epsilon)}=\frac{S_{T}^{(\epsilon)}-S_{T}^{(0)}}{\epsilon}$ can be expressed as

$$
f_{G^{(\epsilon)}}(x) \sim f_{g_{1 T}}(x)+\epsilon C_{13} H_{3}\left(x ; \Sigma_{T}\right) f_{g_{1 T}}(x)+\cdots
$$

where

with

$$
f_{g_{1 T}}(x)=\frac{1}{\sqrt{2 \pi \Sigma_{T}}} \exp \left(-\frac{x^{2}}{2 \Sigma_{T}}\right)
$$

$$
\Sigma_{T}=\int_{0}^{T}\left(S_{t}^{(0)}\right)^{2 \beta}\left(\sigma_{t}^{(0)}\right)^{2} d t
$$


and

$$
\begin{aligned}
C_{13}= & \frac{1}{\Sigma_{T}^{3}} \int_{0}^{T} \int_{0}^{t_{1}} \beta\left(S_{t_{1}}^{(0)}\right)^{2 \beta-1}\left(\sigma_{t_{1}}^{(0)}\right)^{2}\left(S_{t_{2}}^{(0)}\right)^{2 \beta}\left(\sigma_{t_{2}}^{(0)}\right)^{2} d t_{2} d t_{1} \\
& +\frac{1}{\Sigma_{T}^{3}} \int_{0}^{T} \int_{0}^{t_{1}} e^{-\lambda\left(t_{1}-t_{2}\right)}\left(S_{t_{1}}^{(0)}\right)^{2 \beta} \sigma_{t_{1}}^{(0)} \nu_{1}\left(S_{t_{2}}^{(0)}\right)^{\beta}\left(\sigma_{t_{2}}^{(0)}\right)^{2} d t_{2} d t_{1} .
\end{aligned}
$$

Note also that $C_{13}$ is calculated in closed form; the expression is omitted, which is available upon request. Moreover, by a similar calculation to that in Section 3.2 , an approximate price of a call option on $S^{(\epsilon)}$ at time 0 with maturity $T$ and strike $K=S_{T}^{(0)}-\epsilon y$ up to $\epsilon^{2}$-order is given by

$$
\begin{aligned}
C(K, T)= & \epsilon P(0, T)\left(\Sigma_{T} f_{g_{1 T}}(y)+y N\left(\frac{y}{\sqrt{\Sigma_{T}}}\right)\right) \\
& -\epsilon^{2} P(0, T) C_{13} \Sigma_{T}^{2} y f_{g_{1 T}}(y)+o\left(\epsilon^{2}\right)
\end{aligned}
$$

where $N(\cdot)$ is a cumulative distribution function of the standard normal distribution. Higher-order asymptotic expansions can be calculated in a similar manner.

\subsubsection{Numerical Example: $\lambda=0$ (SABR case)}

First, we consider European plain-vanilla call and put prices under the original SABR case $(\lambda=0$ in the $\lambda$-SABR model). We calculate approximated prices by the asymptotic expansion method up to the fifth order. Note that all the solutions to the differential equations are obtained in closed form. Thus, the computation is very fast(e.g. the computation time is within $10^{-5} \sim 10^{-6}$ second for the fifth-order expansion). We also calculate approximated prices by Hagan et al.[7] to compare accuracy of its approximation with ours. Benchmark values are computed by Monte Carlo simulations. In the simulations for the benchmark values, we use Euler-Maruyama scheme as a discretization scheme with 1024 time steps, and generate $10^{8}$ paths in each simulation. $\epsilon$ is set to be one and other parameters used in the test are given in Table 1.

Table 1: Parameters used in the SABR $(\lambda=0)$ case

\begin{tabular}{|c|c|c|c|c|c|c|}
\hline Parameter & $S(0)$ & $\beta$ & $\sigma(0)$ & $\nu$ & $\rho$ & $T$ \\
\hline \hline $\mathrm{i}$ & 100 & 0.5 & 3.0 & 0.3 & -0.7 & 10 \\
\hline
\end{tabular}

Results are in Table 3 and Figure 1. From the results, we can see that the higher-order asymptotic expansion almost always improves accuracy of the approximation by the lower ones. While sometimes the third-order approximation does not perform well, particularly in OTM options, the fifth-order one approximates the prices almost perfectly in these settings. This strongly supports importance of computing high-order terms, and hence of our method. We also see the fifth-order expansion has equal or smaller approximation errors than Hagan et al.[7]'s formula. Moreover, as seen in the next example, the asymptotic expansion method can be easily extend to the $\lambda$-SABR $(\lambda \neq 0)$ case.

\subsubsection{Numerical Example: $\lambda \neq 0$}

Next, we consider the European option prices under the $\lambda$-SABR model with $\lambda \neq 0$. Parameters used in the test are given in Table 2 (and $\epsilon=1$ as well as in the previous examples). 
Table 2: Parameters used in the $\lambda$-SABR $(\lambda \neq 0)$ case

\begin{tabular}{|c|c|c|c|c|c|c|c|c|}
\hline Parameter & $S(0)$ & $\beta$ & $\sigma(0)$ & $\lambda$ & $\theta$ & $\nu$ & $\rho$ & $T$ \\
\hline ii & 100 & 0.5 & 3.0 & 0.1 & 3.0 & 0.3 & -0.7 & 10 \\
\hline iii & 100 & 1.0 & 0.3 & 0.1 & 0.3 & 0.3 & -0.7 & 10 \\
\hline
\end{tabular}

We calculate approximated prices by the asymptotic expansion method up to the fifth order. Note that all the solutions to the differential equations are obtained analytically. Further, for the case of $\beta=1$ in the $\lambda$-SABR model (case iii), we can also apply the log-normal asymptotic expansion method given in Section 3.3. This gives the slightly different approximation formula from that with the normal asymptotic expansion method. Note also that the system of ODEs appearing in the log-normal expansion formula are solved analytically as in the normal asymptotic expansion case. We calculate approximated prices by the log-normal asymptotic expansion up to the forth order. We also calculate option prices by Hagan et al.[7]'s formula by setting $\lambda=0$ in the model which can be thought as the SABR approximation to the $\lambda$-SABR model. Benchmark prices are computed by Monte Carlo simulations with Euler-Maruyama discretization scheme with 1024 time steps, and we generate $10^{8}$ paths in each simulation.

Results for the normal asymptotic expansion are in Table 3 and Figure 2 and 3 , and results for the log-normal expansion for case iii are in Table 4 and Figure 4. Note that the 0th-order log-normal expansion (indicated by 'LogNormal' in Table 4 and Figure 4) gives a simple log-normal approximation of the model.

From the results, in each case, as well as the examples in the original SABR model the higher-order expansion or log-normal expansion almost always improve accuracy of the approximation by the lower-order expansions. On the other hand, a naive application of Hagan et al.[7]'s formula to $\lambda$-SABR $\operatorname{model}(\lambda \neq 0)$ seems to fail to capture the underlying distribution and the resulting option prices. This might be caused by the fact that it cannot be directly applied to the $\lambda$-SABR setting while our method is applicable to a general setting in the unified manner. Further, unlike Hagan et al.[7]'s one whose high-order expansions are difficult to calculate, our method easily provides us the approximation with an arbitrary-high order as we have already seen. These results support flexibility of ours in financial practice.

In addition, for SABR and $\lambda$-SABR models we compare computation times of our method with the ones of the method by Hagan et al. [7]. As the computation times of both methods are very fast $\left(10^{-5} \sim 10^{-6}\right.$ second per option), we implement 10,000 times calculations of 20 options with different strike prices for comparison. Then, the computation times are of the same order for both methods: the ratios of the times based on our method relative to the ones by Hagan et al. [7] are approximately $0.3 \sim 1.6$ for the cases in which both methods achieve the similar accuracies.

At the end of this section, we stress the applicability of our high-order method to much higher-dimensional settings. In fact, in [26], [27], and [28] we apply this technique to pricing options on foreign exchange rates with a stochastic volatility and Libor market models of domestic/foreign interest rates; the model consists of ten underlying stochastic variables. Even in this highdimensional model, our proposed scheme performs very well for valuation of options with long maturities such as fifteen years. See those papers for the detail. 


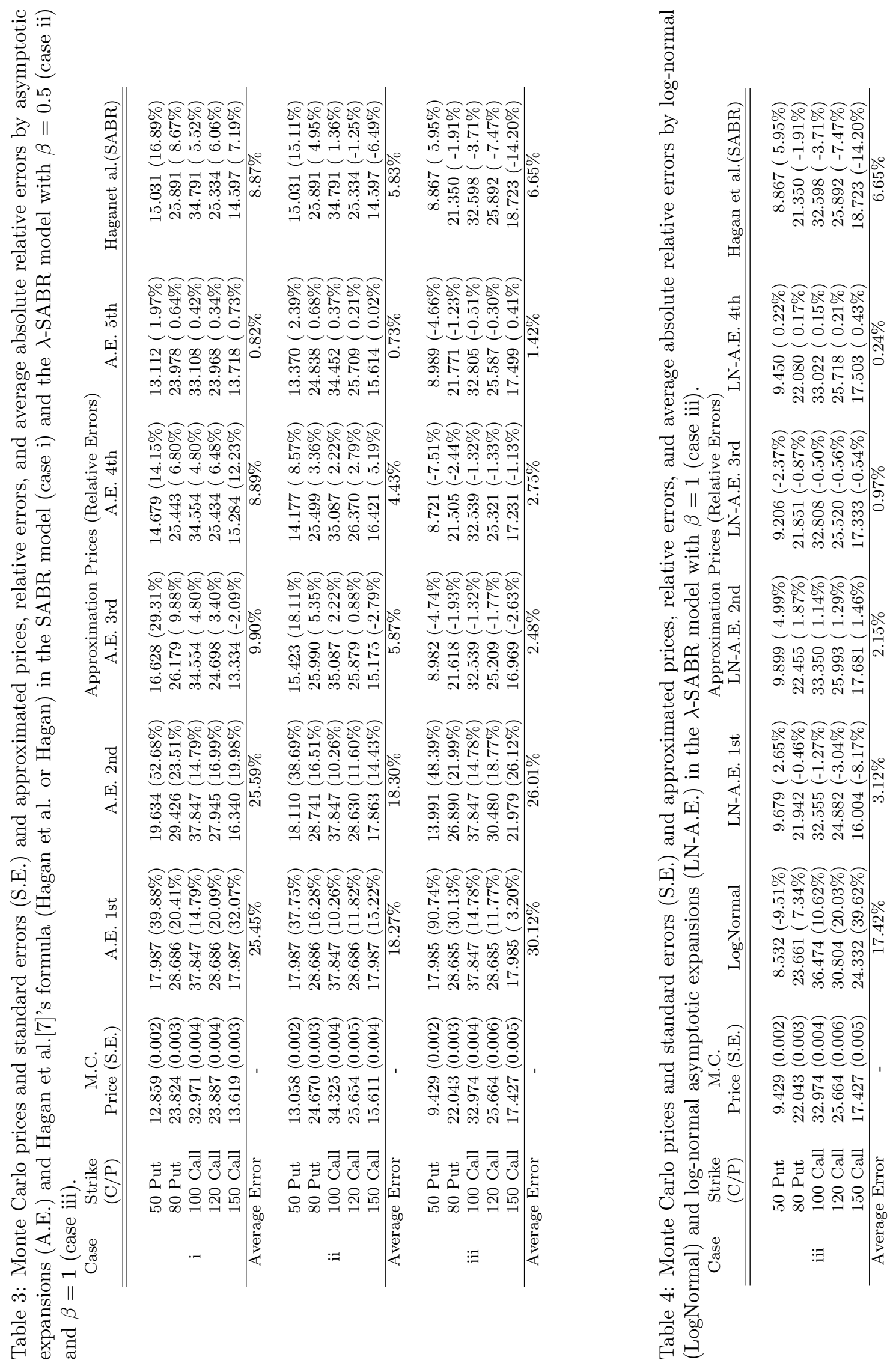



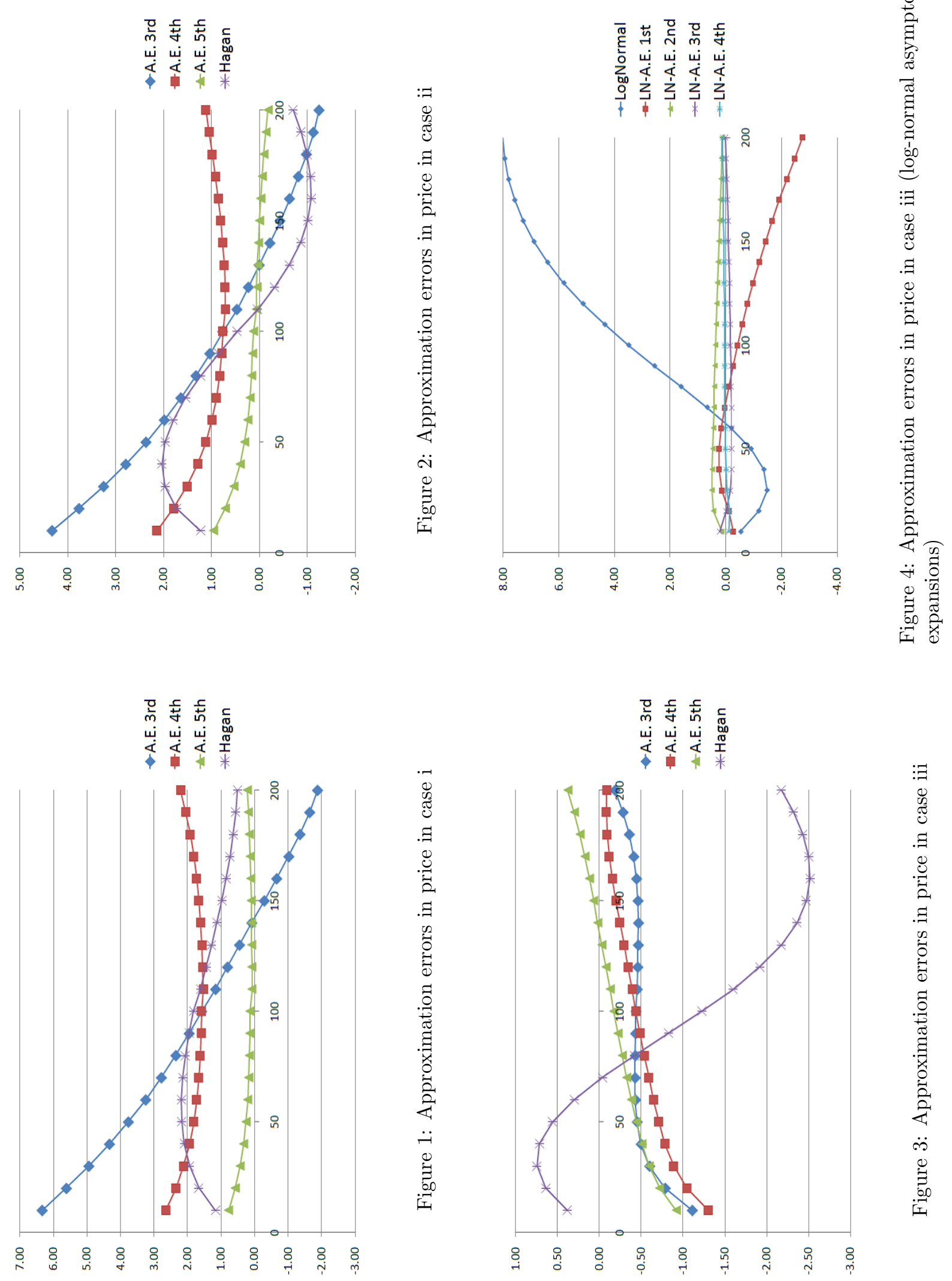


\section{References}

[1] Antonov, A. and Misirpashaev, T.[2009], "Projection on a Quadratic Model by Asymptotic Expansion with an Application to LMM Swaption," Working Paper.

[2] Andersen, L.B.G. and Hutchings, N.A.[2009], "Parameter Averaging of Quadratic SDEs With Stochastic Volatility," Working Paper.

[3] Ben Arous, G., Laurence, P.[2009], "Second order expansion for implied volatility in two factor local stochastic volatility models and applications to the dynamic $\lambda$-SABR model," Working Paper.

[4] Fouque, J.-P., Papanicolaou, G. and Sircar, K. R.[1999], "Financial Modeling in a Fast Mean-reverting Stochastic Volatility Environment," AsiaPacific Financial Markets, Vol. 6(1), pp.37-48.

[5] Fouque, J.-P., Papanicolaou, G. and Sircar, K. R.[2000], Derivatives in financial Markets with Stochastic Volatility, Cambridge University Press.

[6] Gatheral, J., Hsu, E.P., Laurence, P., Ouyang, C. and Wang, T-H.[2009], "Asymptotics of implied volatility in local volatility models," Working Paper.

[7] Hagan, P.S., Kumar, D., Lesniewski, A.S., and Woodward, D.E. [2002], "Managing smile risk," Willmott Magazine, 15, 84-108.

[8] Ikeda, N. and Watanabe, S. [1989], Stochastic Differential Equations and Diffusion Processes, Second Edition, North-Holland/Kodansha, Tokyo.

[9] Kunitomo, N. and Takahashi, A. [1992], "Pricing Average Options," Japan Financial Review, Vol. 14, 1-20. (in Japanese).

[10] Kunitomo, N. and Takahashi, A. [2001], "The Asymptotic Expansion Approach to the Valuation of Interest Rate Contingent Claims," Mathematical Finance, Vol. 11, 117-151.

[11] Kunitomo, N. and Takahashi, A. [2003a], "On Validity of the Asymptotic Expansion Approach in Contingent Claim Analysis," Annals of Applied Probability Vol. 13-3, 914-952.

[12] Labordere, P.H. [2005a], "A General Asymptotic Implied Volatility for Stochastic Volatility Models", cond-mat/0504317.

[13] Labordere, P.H. [2005b], "Solvable Local and Stochastic Volatility Models: Supersymmetric Methods in Option Pricing," Working Paper.

[14] Matsuoka, R. Takahashi, A. and Uchida, Y. [2004], "A New Computational Scheme for Computing Greeks by the Asymptotic Expansion Approach," Asia-Pacific Financial Markets, Vol.11, 393-430.

[15] Muroi, Y. [2005], "Pricing Contingent Claims with Credit Risk: Asymptotic Expansion Approach," Finance and Stochastics, Vol. 9(3), 415-427.

[16] Nualart, D. [1995], "The Malliavin Calculus and Related Topics," Springer.

[17] Nualart, D., Üstünel A. S. and Zakai M. [1988], "On the moments of a multiple Wiener-Itô integral and the space induced by the polynomials of the integral," Stochastics, Vol. 25, 233-340. 
[18] Rubinstein, M.[1983], "Displaced Diffusion Option Pricing," Journal of Finance, Vol.38-1.

[19] Siopacha, M. and Teichmann, J.[2007], "Weak and Strong Taylor Methods for Numerical Solutions of Stochastic Differential Equations," Working paper.

[20] Takahashi, A. [1995], "Essays on the Valuation Problems of Contingent Claims," Unpublished Ph.D. Dissertation, Haas School of Business, University of California, Berkeley.

[21] Takahashi, A. [1999], "An Asymptotic Expansion Approach to Pricing Contingent Claims," Asia-Pacific Financial Markets, Vol. 6, 115-151.

[22] Takahashi, A. [2009], "On an Asymptotic Expansion Approach to Numerical Problems in Finance," Selected Papers on Probability and Statistics, pp. 199-217, 2009, American Mathematical Society.

[23] Takahashi, A. and Takehara, K.[2007], "An Asymptotic Expansion Approach to Currency Options with a Market Model of Interest Rates under Stochastic Volatility Processes of Spot Exchange Rates," Asia-Pacific Financial Markets, Vol.14, pp. 69-121.

[24] Takahashi, A. and Takehara, K.[2008a], "Fourier Transform Method with an Asymptotic Expansion Approach: an Applications to Currency Options," International Journal of Theoretical and Applied Finance, Vol. 11(4), pp. 381-401.

[25] Takahashi, A. and Takehara, K. [2008b], "A Hybrid Asymptotic Expansion Scheme: an Application to Currency Options," Working paper, CARF-F-116, the University of Tokyo, http://www.carf.e.utokyo.ac.jp/workingpaper/

[26] Takahashi, A., Takehara, K. and Toda, M. [2009], "Computation in an Asymptotic Expansion Method," Working paper, CARF-F-149, the University of Tokyo, http://www.carf.e.u-tokyo.ac.jp/workingpaper/

[27] Takehara, K., Takahashi, A. and Masashi Toda [2010], "New Unified Computation Algorithm in a High-Order Asymptotic Expansion Scheme," Recent Advances in Financial Engineering (the Proceedings of KIER-TMU International Workshop on Financial Engineering 2009), pp. 231-251.

[28] Takehara, K., Toda, M. and Takahashi, A. [2011], "Application of a HighOrder Asymptotic Expansion Scheme to Long-Term Currency Options," International Journal of Business and Finance Research, vol. 5-3, pp. 87100.

[29] Takahashi, A. and Yamada, T. [2009], "An Asymptotic Expansion with Push Down Malliavin Weights," Working paper, CARF-F-194, the University of Tokyo, http://www.carf.e.u-tokyo.ac.jp/workingpaper/

[30] Takahashi, A. and Yoshida, N. [2004], "An Asymptotic Expansion Scheme for Optimal Investment Problems," Statistical Inference for Stochastic Processes, Vol. 7-2, 153-188.

[31] Takahashi, A. and Yoshida, N. [2005], "Monte Carlo Simulation with Asymptotic Method," The Journal of Japan Statistical Society, Vol. 35$2,171-203$. 
[32] Watanabe, S. [1987], "Analysis of Wiener Functionals (Malliavin Calculus) and its Applications to Heat Kernels," The Annals of Probability, Vol. 15, $1-39$.

[33] Yoshida, N. [1992a], "Asymptotic Expansion for Small Diffusions via the Theory of Malliavin-Watanabe," Probability Theory and Related Fields, Vol. 92, 275-311.

[34] Yoshida, N. [1992b], "Asymptotic Expansions for Statistics Related to Small Diffusions," The Journal of Japan Statistical Society, Vol. 22, 139159 .

\section{Appendix}

\subsection{Proof of Lemma 1 in Section 3}

Since the system of Hermite polynomials $\left\{H_{n}(x ; \Sigma)\right\}$ is an orthogonal basis of $L^{2}(\mathbf{R}, \mu)$, and $E[X \mid Z=x] \in L^{2}(\mathbf{R}, \mu)$, we have the following unique expansion of $E[X \mid Z=x]$ in $L^{2}(\mathbf{R}, \mu)$ :

$$
E[X \mid Z=x]=\sum_{n=0}^{\infty} \frac{a_{n}}{\Sigma^{n}} H_{n}(x ; \Sigma) .
$$

Since we have another Taylor expansion

$$
e^{i \xi x}=e^{-\frac{\xi^{2}}{2} \Sigma} \sum_{n=0}^{\infty} \frac{H_{n}(x ; \Sigma)}{n !}(i \xi)^{n},
$$

then,

$$
\begin{aligned}
e^{\frac{\xi^{2}}{2} \Sigma} \mathbf{E}\left[e^{i \xi Z} X\right] & =e^{\frac{\xi^{2}}{2} \Sigma} \int_{\mathbf{R}} e^{i \xi x} \mathbf{E}[X \mid Z=x] \mu(d x) \\
& =\int_{\mathbf{R}} \sum_{m=0}^{\infty} \frac{H_{m}(x ; \Sigma)}{m !}(i \xi)^{m} \sum_{n=0}^{\infty} a_{n} H_{n}(x ; \Sigma) \mu(d x) \\
& =\sum_{n=0}^{\infty} a_{n}(i \Sigma)^{n} \xi^{n}
\end{aligned}
$$

Comparing to the coefficients of the Taylor series of $e^{\frac{\xi^{2}}{2} \Sigma} \mathbf{E}\left[e^{i \xi Z} X\right]$ around 0 with respect to $\xi$, we see that $a_{n}$ can be written as (9). 


\subsection{Proof of Theorem 1 in Section 3.1}

First, Applying Itô's formula to $\left(\prod_{j=1}^{\beta} A_{l_{j} t}^{d_{j}}\right)$, we have

$$
\begin{aligned}
& d\left(\prod_{j=1}^{\beta} A_{l_{j} t}^{d_{j}}\right)=\sum_{k=1}^{\beta}\left(\prod_{\substack{j=1 \\
j \neq k}}^{\beta} A_{l_{j} t}^{d_{j}}\right) d A_{l_{k} t}^{d_{k}}+\sum_{\substack{k, m=1 \\
k<m}}^{\beta}\left(\prod_{\substack{j=1 \\
j \neq k, m}}^{\beta} A_{l_{j} t}^{d_{j}}\right) d\left\langle A_{l_{k}}^{d_{k}}, A_{l_{m}}^{d_{m}}\right\rangle_{t} \\
& =\sum_{k=1}^{\beta}\left(\prod_{\substack{j=1 \\
j \neq k}}^{\beta} A_{l_{j} t}^{d_{j}}\right) \frac{1}{l_{k} !} \partial_{\epsilon}^{l_{k}} V_{0}^{d_{k}}\left(X_{t}^{(0)}, 0\right) d t \\
& +\sum_{k=1}^{\beta} \sum_{l=1}^{l_{k}}\left(\prod_{\substack{j=1 \\
j \neq k}}^{\beta} A_{l_{j} t}^{d_{j} t} \sum_{\vec{m}_{\gamma}, \overrightarrow{\vec{d}}_{\gamma}}^{(l)} \frac{1}{\left(l_{k}-l\right) !} \frac{1}{\gamma !}\left(\prod_{j^{\prime}=1}^{\gamma} A_{m_{j^{\prime}} t}^{\tilde{d}_{j^{\prime}}}\right) \partial_{\tilde{d}_{\gamma}}^{\gamma} \partial_{\epsilon}^{l_{k}-l} V_{0}^{d_{k}}\left(X_{t}^{(0)}, 0\right) d t\right. \\
& +\sum_{k=1}^{\beta}\left(\prod_{\substack{j=1 \\
j \neq k}}^{\beta} A_{l_{j} t}^{d_{j}}\right) \sum_{\vec{m}_{\gamma}, \overrightarrow{\tilde{d}}_{\gamma}}^{\left(l_{k}-1\right)} \frac{1}{\gamma !}\left(\prod_{j^{\prime}=1}^{\gamma} A_{m_{j^{\prime}} t}^{\tilde{d}_{j^{\prime}}}\right) \partial_{\tilde{d}_{\gamma}}^{\gamma} V^{d_{k}}\left(X_{t}^{(0)}\right) d W_{t} \\
& +\sum_{\substack{k, m=1 \\
k<m}}^{\beta}\left(\prod_{\substack{j=1 \\
j \neq k, m}}^{\beta} A_{l_{j} t}^{d_{j}}\right) \sum_{\vec{m}_{\gamma}, \overrightarrow{\tilde{d}}_{\gamma}}^{\left(l_{k}-1\right)} \sum_{\vec{m}_{\delta}, \overrightarrow{\hat{d}}_{\delta}}^{\left(l_{m}-1\right)} \frac{1}{\gamma ! \delta !} \\
& \times\left(\prod_{j^{\prime}=1}^{\gamma} A_{m_{j^{\prime}}}^{\tilde{d}_{j^{\prime}}}\right) \partial_{\tilde{d}_{\gamma}}^{\gamma} V^{d_{k}}\left(X_{t}^{(0)}\right)\left(\prod_{j^{\prime}=1}^{\delta} A_{m_{j^{\prime}} t}^{\hat{d}_{j^{\prime}}}\right) \partial_{\hat{d}_{\delta}}^{\delta} V^{d_{m}}\left(X_{t}^{(0)}\right) d t .
\end{aligned}
$$

Note also that

$$
d Z_{t}^{\langle\xi\rangle}=(i \xi) \hat{V}\left(X_{t}^{(0)}, t\right) Z_{t}^{\langle\xi\rangle} d W_{t} .
$$

Then, applying Itô's formula again to $\left(\prod_{j=1}^{\beta} A_{l_{j} t}^{d_{j}} Z_{t}^{\langle\xi\rangle}\right)$ and taking expectations on both sides, we obtain the result. 